\title{
Efficient Binary-Level Coverage Analysis
}

\author{
M. Ammar Ben Khadra \\ Technische Universität Kaiserslautern \\ Germany \\ khadra@eit.uni-kl.de
}

\author{
Dominik Stoffel \\ Technische Universität Kaiserslautern \\ Germany \\ stoffel@eit.uni-kl.de
}

\author{
Wolfgang Kunz \\ Technische Universität Kaiserslautern \\ Germany \\ kunz@eit.uni-kl.de
}

\begin{abstract}
Code coverage analysis plays an important role in the software testing process. More recently, the remarkable effectiveness of coverage feedback has triggered a broad interest in feedback-guided fuzzing. In this work, we introduce bcov, a tool for binary-level coverage analysis. Our tool statically instruments x86-64 binaries in the ELF format without compiler support. We implement several techniques to improve efficiency and scale to large real-world software. First, we bring Agrawal's probe pruning technique to binary-level instrumentation and effectively leverage its superblocks to reduce overhead. Second, we introduce sliced microexecution, a robust technique for jump table analysis which improves $\mathrm{CFG}$ precision and enables us to instrument jump table entries. Additionally, smaller instructions in x86-64 pose a challenge for inserting detours. To address this challenge, we aggressively exploit padding bytes and systematically host detours in neighboring basic blocks.

We evaluate bcov on a corpus of 95 binaries compiled from eight popular and well-tested packages like FFmpeg and LLVM. Two instrumentation policies, with different edge-level precision, are used to patch all functions in this corpus - over 1.6 million functions. Our precise policy has average performance and memory overheads of $14 \%$ and $22 \%$ respectively. Instrumented binaries do not introduce any test regressions. The reported coverage is highly accurate with an average F-score of $99.86 \%$. Finally, our jump table analysis is comparable to that of IDA Pro on gcc binaries and outperforms it on clang binaries.
\end{abstract}

\section{CCS CONCEPTS}

- Software and its engineering $\rightarrow$ Software testing and debugging; • Security and privacy $\rightarrow$ Software reverse engineering.

\section{KEYWORDS}

code coverage analysis, jump table analysis, binary instrumentation

\section{ACM Reference Format:}

M. Ammar Ben Khadra, Dominik Stoffel, and Wolfgang Kunz. 2020. Efficient Binary-Level Coverage Analysis. In Proceedings of the 28th ACM foint European Software Engineering Conference and Symposium on the Foundations of Software Engineering (ESEC/FSE '20), November 8-13, 2020, Virtual Event, USA. ACM, New York, NY, USA, 12 pages. https://doi.org/10.1145/3368089. 3409694

Permission to make digital or hard copies of all or part of this work for personal or classroom use is granted without fee provided that copies are not made or distributed for profit or commercial advantage and that copies bear this notice and the full citation on the first page. Copyrights for components of this work owned by others than ACM must be honored. Abstracting with credit is permitted. To copy otherwise, or republish, to post on servers or to redistribute to lists, requires prior specific permission and/or a fee. Request permissions from permissions@acm.org.

ESEC/FSE '20, November 8-13, 2020, Virtual Event, USA

(C) 2020 Association for Computing Machinery.

ACM ISBN 978-1-4503-7043-1/20/11 . \$15.00

https://doi.org/10.1145/3368089.3409694

\section{INTRODUCTION}

Code coverage analysis is commonly used throughout the software testing process [2]. Structural coverage metrics such as statement and branch coverage can inspire confidence in a program under test (PUT), or at least identify untested code [20,21]. Additionally, coverage analysis has demonstrated its usefulness in test suite reduction [42], fault localization [31], and detection of compiler bugs [27]. Moreover, certain coverage requirements are mandated by the standards in safety-critical domains $[16,22]$.

In recent years, feedback-guided fuzzing has emerged as a successful method for automatically discovering software bugs and security vulnerabilities [8, 33, 35, 43]. Notably, AFL [44] has pioneered the usage of code overage as a generic and effective feedback signal. This success inspired a fuzzing "renaissance" and helped move fuzzing to industrial-scale adoption like in Google's OSS-Fuzz [30].

In this work, we introduce bcov, a tool for binary-level coverage analysis using static instrumentation. bcov works directly on x86-64 binaries in the ELF format without compiler support. It implements a trampoline-based approach where it inserts probes in targeted locations to track basic block coverage. Each probe consists of a detour that diverts control flow to a designated trampoline. The latter updates coverage data using a single pc-relative mov instruction, potentially executes relocated instructions, and then restores control flow to its original state. Making this scheme to work efficiently and transparently on large and well-tested $\mathrm{C}$ and $\mathrm{C}++$ programs required addressing several challenges:

Probe pruning $(\S 3)$. Instrumenting all basic blocks (BBs) can be inefficient, or even impossible, in x86-64 ISA due to its instructionsize variability. We adopt the probe pruning technique proposed by Agrawal [1] where dominator relationships between BBs are used to group them in superblocks (SBs). SBs are arranged in a superblock dominator graph. Covering a single BB implies that all BBs in the same SB are also covered, in addition to SBs dominating the current SB. This allows us to significantly reduce the instrumentation overhead and size of coverage data.

Precise CFG analysis ( $\$ 4)$. Imprecision in the recovered control flow graph (CFG) can cause false positives in the reported coverage. It can also cause instrumentation errors which lead to crashes in a PUT. To address this challenge, we propose sliced microexecution, a precise and robust technique for jump table analysis. Also, we implement a non-return analysis that eliminates spurious CFG edges after non-return calls. Our experiments show that bcov can outperform IDA Pro, the leading industry disassembler.

Static instrumentation ( $(5)$. Given a set of BBs in an SB, we need to choose the best $\mathrm{BB}$ to probe based on the expected overhead of restoring control flow. We make this choice using a classification of BBs in x86-64 into 9 types. Also, some BBs can be too short to insert a detour. Their size is less than 5 bytes. We address this 


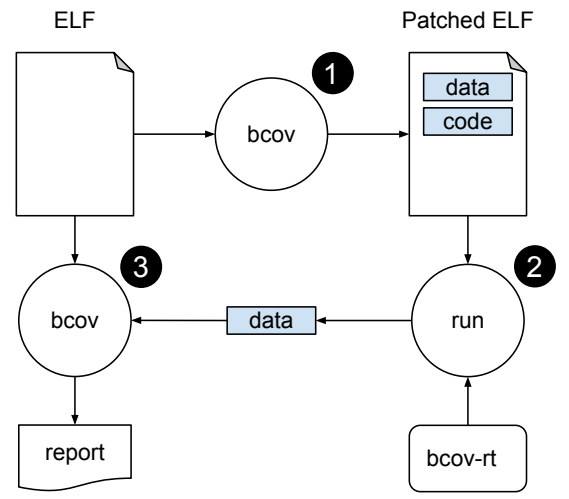

Figure 1: The general workflow of bcov. A binary is patched with extra code segment (trampolines) and data segment (coverage data). Our bcov-rt library dumps the data segment at run-time. In our prototype, reporting coverage requires re-analyzing the binary.

challenge by (1) aggressively exploiting padding bytes, (2) instrumenting jump table entries, and (3) introducing a greedy strategy for detour hosting where a larger $\mathrm{BB}$ can host the detour of a neighboring short $\mathrm{BB}$. Combining these techniques with probe pruning enables tracking coverage of virtually all BBs.

\subsection{Design Overview}

Figure 1 depicts the workflow of bcov. Given an ELF module as input, bcov first analyzes module-level artifacts, such as the call graph, before moving to function-level analyses to build the CFG and dominator graphs. Then, bcov will choose appropriate probe locations and estimate the required code and data sizes depending on the instrumentation policy chosen by the user. Our prototype supports two instrumentation policies. The first is a complete coverage policy where for any test input it is possible to precisely identify covered BBs. The second one is a heuristic coverage policy where we probe only the leaf SBs in the superblock dominator graph. Running a test suite that covers all leaf SBs implies that $100 \%$ code coverage is reached. We refer to these policies as any-node and leaf-node policies respectively. On average, the any-node policy probes $46 \%$ of BBs compared to $30 \%$ in the leaf-node policy. Average performance overheads are $14 \%$ and $8 \%$ respectively.

The patching phase can start after completing the previous analysis phase. Here, bcov first extends the ELF module by allocating two loadable segments: a code segment where trampolines are written and a data segment for storing coverage data. Then, bcov iterates over all probes identified by the chosen instrumentation policy. Each probe represents a single SB. Generally, patching a probe requires inserting a detour targeting its corresponding trampoline. The detour can be a pc-relative jmp or call instruction. The trampoline first updates coverage data and then restores control flow to its state in the original module as depicted in Figure 2.

The data segment has a simple format consisting of a small header and a byte array that is initialized to zeros. Setting a byte to one indicates that its corresponding $\mathrm{SB}$ is covered. It is trivial to compress this data on disk as only the LSB of each byte is used. For example, this enables storing complete coverage data of 1lc (LLVM

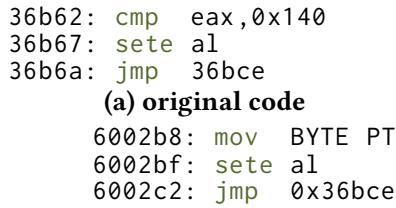

(c) trampoline

Figure 2: bcov patching example. (a) instruction at 0x36b67 must be relocated as the size of jump at 0x36b6a is only two bytes. (b) relocated instructions are replaced with a 5 byte detour at $0 \times 36$ b67. (c) coverage update happens at $0 \times 6002 b 8$. Control flow is then restored after executing the relocated instruction at $0 \times 6002 \mathrm{bf}$.

backend) in $65 \mathrm{~KB}$ only. ${ }^{1}$ Our data format also enables merging coverage data of multiple tests using a simple bitwise OR operation.

Dumping coverage data requires linking against bcov-rt, our small runtime library. Alternatively, bcov-rt can be injected using the LD_PRELOAD mechanism to avoid modifying the build system. Coverage data can be dumped on process shutdown or upon receiving a user signal. The latter enables online coverage tracking of long-running processes. Note that the data segment starts with a magic number which allows bcov-rt to identify it.

This design makes bcov achieve three main goals, namely, transparency, performance, and flexibility. Program transparency is achieved by not modifying program stack, heap, nor any generalpurpose register. Also, coverage update requires a single pc-relative mov instruction which has a modest performance overhead. Finally, bcov works directly on the binary without compiler support and largely without changes to the build system. This enables users to flexibly adapt their instrumentation policy without recompilation.

To summarize, we make the following key contributions:

- We are the first to bring Agrawal's probe pruning technique to binary-level instrumentation. We show that its superblocks can be effectively leveraged to optimize probe selection and reduce coverage data.

- We introduce sliced microexecution, a robust method for jump table analysis. It significantly improves CFG precision and allows us to instrument jump table entries.

- We significantly push the state of the art in trampoline-based static instrumentation and show that it can be used to track code coverage efficiently and transparently.

We implemented our contributions in the tool bcov, which we make publicly available: https://doi.org/10.5281/zenodo.3876047

We extensively experimented with bcov. In this respect, we selected 8 popular and well-tested subjects such as ffmpeg and llc. We compiled them using 4 recent major versions of gcc and clang at 3 different optimization levels each. In total, we used bcov to instrument 95 binaries and more than 1.6 million functions. Instrumented binaries did not introduce any test regressions.

\section{MOTIVATION}

There is a plethora of tools dedicated to coverage analysis. They vary widely in terms of goals and features. Therefore, we motivate the need for our approach via a comparison with a representative set of popular tools. Our discussion is based on Table 1.

\footnotetext{
${ }^{1}$ The binary has around $1 \times 10^{6} \mathrm{BBs}$ which contain more than $4 \times 10^{6}$ instructions.
} 
Table 1: A comparison with representative coverage analysis tools. Compiler-dependent tools require modifying the build system and recompilation which limits flexibility. The usability of binary-level tools in the testing workflow is limited. In contrast, bcov only requires replacing a binary with an instrumented version.

\begin{tabular}{lcccccc} 
& & & & \\
& 4 & 0 & 0 & 0 & & \\
gcov & source & complete & $\times$ & $\times$ & $\times$ & $\checkmark$ \\
Ilvm-cov & source & complete & $\times$ & $\checkmark$ & $\times$ & $\checkmark$ \\
sancov & IR & heuristic & $\times$ & $\mathrm{n} / \mathrm{a}$ & $\times$ & $\checkmark$ \\
Intel PT & binary & heuristic & $\checkmark$ & $\checkmark$ & $\times$ & $\times$ \\
drcov & binary & both & $\checkmark$ & $\times$ & $\checkmark$ & $\times$ \\
bcov & binary & both & $\checkmark$ & $\checkmark$ & $\checkmark$ & $\checkmark$ \\
\hline
\end{tabular}

We start with source-level tools supported in gcc and clang, which are gcov and Ilvm-cov respectively. Both track similar artifacts such as statement coverage. The key difference is in the performance of instrumented binaries. gcov can not accurately track code coverage in optimized builds. In comparison, Ilvm-cov features a custom mapping format embedded in LLVM's intermediate representation (IR). This allows it to cope better with compiler optimizations. Also, this mapping format tracks source code regions with better precision compared to gcov.

The ability of a binary-level tool such as bcov to report sourcelevel artifacts is limited by the binary-to-source mapping available. Off-the-shelf debug information can be used to report statement coverage - the most important artifact in practice [20, 23]. In this setting, bcov offers several advantages including: (1) detailed view of individual branch decisions regardless of the optimization level, (2) precise handling of non-local control flow such as longjmp and $\mathrm{C}++$ exception handling, and (3) flexibility in instrumenting only a selected set of functions, e.g., the ones affected by recent changes, which is important for the efficiency of continuous testing [23].

The recent fuzzing renaissance has motivated the need to improve efficiency by heuristically tracking coverage. SanitizerCoverage (sancov) [34] is a pass built into LLVM which supports collecting various types of feedback signals including basic block coverage. It is used in prominent fuzzers like LibFuzzer [28] and Honggfuzz [37]. The performance overhead of sancov is not directly measurable as the usage model varies significantly between sancov users. Also, sancov is tightly coupled with LLVM sanitizers (e.g., ASan) which add varying overhead. Extending bcov with additional feedback signals, similar to sancov, is an interesting future work.

Hardware instruction tracing mechanisms, like Intel ${ }^{\circledR}$ PT (IPT), can also be used for coverage analysis. However, IPT can dump gigabytes of compressed trace data within seconds which can be inefficient to store and post-process. In our experiments, IPT dumped 6.5 GB trace data for a libxerces test that lasted only 5 seconds. Post-processing and deduplication took more than 3 hours. In comparison, our tool can produce an accurate coverage report for the same test after processing a $53 \mathrm{~KB}$ dump in a few seconds. Schumilo et al. [35] propose to heuristically summarize IPT data on the fly and thus avoid storing the complete trace.
Dynamic binary instrumentation (DBI) tools can report binarylevel coverage using dedicated clients (plug-ins) like drcov. DBI tools act as a process virtual machine that JIT-emits instructions to a designated code cache. This process is complex and may break binaries. Moreover, JIT optimizations add overhead to the whole program even if we are only interested in a selected part such as a shared library. Our evaluation includes a comparison with the popular DBI tools Pin [32] and DynamoRIO [9].

\section{PROBE PRUNING}

We provide here the necessary background on the probe pruning techniques implemented in bcov based on Agrawal [1]. The original work considered source-level pruning but only for $C$ programs.

Given a function $F$ with a set of basic blocks $B$ connected in a CFG. The straightforward way to obtain complete coverage data is to probe every basic block $b b \in B$. However, it is possible to significantly reduce the number of required probes by computing dominance relationships between basic blocks in a CFG. We say that $b b_{i}$ predominates $b b_{j}, b b_{i} \stackrel{\text { pre }}{\longrightarrow} b b_{j}$, iff every path from function entry $(E N)$ to $b b_{j}$ goes through $b b_{i}$. Similarly, $b b_{i}$ postdominates $b b_{j}, b b_{i} \stackrel{\text { post }}{\longrightarrow} b b_{j}$, iff every path from $b b_{j}$ to function exit $(E X)$ goes through $b b_{i}$. We say that $b b_{i}$ dominates $b b_{j}$ iff $b b_{i} \stackrel{\text { pre }}{\longrightarrow} b b_{j} \vee b b_{i} \stackrel{\text { post }}{\longrightarrow} b b_{j}$. The predominator and postdominator relationships are represented by the trees $T_{\text {pre }}$ and $T_{\text {post }}$ respectively. The dominator graph (DG) is a directed graph that captures all dominance relationships. It is obtained by the union of both trees $D G=T_{\text {pre }} \cup T_{\text {post }}$, i.e, by merging edges of both trees.

Given a dominator graph and the fact that a particular $b b$ is covered, this implies that all dominators (predecessors) of $b b$ in DG are also covered. This allows us to avoid probing basic blocks that do not increase our coverage information. However, we are interested in moving a step further by leveraging strongly-connected components (SCCs) in the DG. Each SCC represents a superblock, a set of basic blocks with equivalent coverage information. The superblock dominator graph (SB-DG) is constructed by merging SCCs in the DG. That is, each node SB in SB-DG represents a SCC in the DG. An edge is inserted between $S B_{i}$ and $S B_{j}$ iff $\exists b b \in S B_{i}, \exists b b^{\prime} \in S B_{j}$ where $b b$ dominates $b b^{\prime}$

Constructing a SB-DG has a number of benefits. First, it is a convenient tool to measure the coverage information gained from probing any particular basic block. Second, it enables compressing coverage data by tracking superblocks instead of individual basic blocks. Finally, it provides flexibility in choosing the best basic block to probe in a superblock. We show later in section 5.1 how this flexibility can be leveraged to reduce instrumentation overhead.

We implemented two instrumentation policies in bcov, namely, leaf-node and any-node. We discuss them based on the example depicted in Figure 3. In the leaf-node policy, we instrument only the leaves of the SB-DG. Covering all such leaf nodes implies that all nodes in SB-DG are also covered, i.e., achieving $100 \%$ coverage. However, this coverage percentage is usually infeasible in practice. Nevertheless, leaf nodes still provide high coverage information which makes the leaf-node policy useful to approximate the coverage of a test suite at a relatively low overhead.

Generally, we are also interested in inferring the exact set of covered basic blocks given any test input. This is usually not possible 


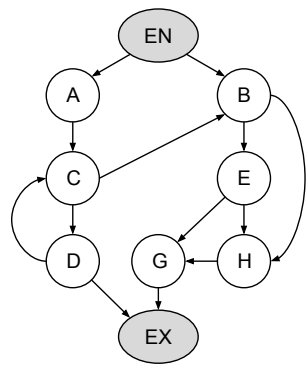

(a) CFG

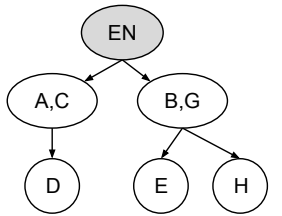

(b) SB-DG graph
Figure 3: An example CFG and its corresponding SB-DG. First, pre-domominator and post-dominator trees are constructed and merged in a dominator graph (DG). SCCs in DG represent nodes in SB-DG. In the leaf-node policy, only leaf nodes in SB-DG, namely, D, $\mathrm{E}$, and $\mathrm{H}$, need to be probed. In the any-node policy, either $\mathrm{A}$ or $\mathrm{C}$ need to be additionally probed. $E N$ and $E X$ are virtual nodes commonly used to simplify dominance analysis.

in the leaf-node policy. For example, given an input that visits the path $A \rightarrow C \rightarrow B \rightarrow H \rightarrow G$, the leaf-node policy can report that the covered set is $\{B, H, G\}$. However, this policy can make no statement about the coverage of $A$ and $C$ since they do not dominate the visited probe in $H$. We address this problem in the any-node policy. The set of superblocks instrumented in this policy is a superset of those in the leaf-node policy. More precisely, $S_{\text {any }}=S_{\text {leaf }} \cup S_{c}$. $S_{c}$ represents the set of critical superblocks in the sense that each $s b \in S_{c}$ can be visited by at least one path in the CFG that does not visit any of its children in the SB-DG.

It is possible to determine $S_{c}$ using an $O(|V|+|E|)$ algorithm where $V$ and $E$ are the nodes and edges in the CFG respectively. We refer to [1] for further details. In Figure 3, the superblock $\{B, G\}$ is non-critical. However, the superblock $\{A, C\}$ is critical and, consequently, will be probed in the any-node policy.

\section{CONTROL FLOW ANALYSIS}

In this section, we first consider the definition of a function at the binary level. Then, we discuss sliced microexecution, our proposed method for jump table analysis.

\subsection{Function Definitions}

The notion of function is important to our approach as it determines the scope of CFG and, consequently, the correctness of dominance relationships. Functions are well-defined constructs in the source code. However, compiler optimizations such as function splitting and inlining significantly change the layout of corresponding binary-level functions.

Fortunately, these optimizations are not of concern to us as long as well-formed function definitions are given to bcov. A function is defined by the pair $F=(s, z)$ where $s$ and $z$ are start address and byte size respectively. A function can have a set of entry and exit points where control flow enters and leaves the function respectively. We say that a function definition is well-formed if (1) its area does not overlap with other functions, and (2) all of its basic blocks are reachable only through its entries.

Definitions source. Our tool uses linker symbols as a source of well-formed function definitions. These symbols, unlike debug symbols, are available by default in all builds. In stripped binaries, bcov can read function definitions from call-frame information (CFI) records which can be found in the .eh_frame section. This section stores the data necessary for stack unwinding and is part of the loadable image of the binary, i.e., is not stripped. These records must be available to enable $\mathrm{C}++$ exception handling. However, they are typically available in $\mathrm{C}$ binaries as well since they are needed for crash reporting, among other tasks.

Note that CFI records might not contain all the functions defined in linker symbols. For example, developers might exclude CFI records of leaf functions to save memory. However, we empirically observed that function definitions in CFI records largely match those found in linker symbols. Additionally, in the unlikely case where CFI records are unavailable, we may still resort to function identification techniques such as [5, 6].

Function entries. The main entry of a function is trivially defined by its start address. Other functions can either call or tail-call only the main entry. We have empirically validated this assumption in our dataset. That is, we have not found any instance where a (direct) function call targets an internal basic block in another function. However, non-local control transfer mechanisms, such as long jmp and exception handling, violate this assumption. We refer to possible targets of non-local control transfer as auxiliary function entries. Such entries are not dominated by, or even unreachable from, the main function entry. Auxiliary entries of longjmp are identified during CFG construction. They are simply the successor of each basic block that calls setjmp.

The identification of auxiliary entries used in exception handling is more elaborate. The Itanium $\mathrm{C}++\mathrm{ABI}$ specifies the exception handling standard used in modern Unix-like systems. Of interest to us in this specification is the landing pad which is a code section responsible for catching, or cleaning up after, an exception. A function can have several landing pads, e.g., it can catch exceptions of different types. We consider each landing pad to be an auxiliary entry. Collecting landing pad addresses requires bcov to iterate over all CFI records in the .eh_frame section. More specifically, bcov examines all Frame Description Entry (FDE) records looking for a pointer to a language-specific data area (LSDA). If such a pointer exists, then bcov parses the corresponding LSDA to extract landing pad addresses.

Function exits. Our tool analyzes the CFG to identify the basic blocks where the control flow leaves a function. We consider two parameters (1) the type of the control-transfer instruction which can be jmp, call, or ret, and (2) whether it is a direct or indirect instruction. A jmp targeting another function is a tail-call and generally also an exit point. However, the jump table analysis discussed in section 4.2 can determine that certain indirect jmp are intra-procedural, i.e., local to the function. On the other hand, a call typically returns, i.e, is not an exit point, except for calls to non-return functions. The non-return analysis implemented in bcov is responsible for identifying such functions. Finally, we consider all ret instructions to be exit points.

Our model of a function occupying a contiguous code region is simple; yet, we found it to be consistent with our large dataset. Moreover, it can be augmented with additional analyses to identify function entries and exits. This provides enough flexibility to handle 
Table 2: Hypotheses tested, or falsified, to analyze a jump table. Backward slicing answers \# 1 to \#3. Microexecution is used to falsify hypotheses and recover the jump table.

\begin{tabular}{ll} 
Hypothesis & Action \\
\hline (1) Depends on constant base address? & $\begin{array}{l}\text { if yes test (2) } \\
\text { else abort }\end{array}$ \\
(2) Is constrained by a bound condition? & $\begin{array}{l}\text { if yes test (3) } \\
\text { else assume (4) }\end{array}$ \\
(3) Bound condition dominates jump table? & $\begin{array}{l}\text { if yes do recovery } \\
\text { else assume (4) }\end{array}$ \\
& do recovery and \\
(4) Assume jump table is data-bounded & try tolsify \\
\hline
\end{tabular}

special situations that might arise in practice, for example, using ret to implement indirect calls in Retpoline [39].

\subsection{Jump Table Analysis}

Recovering the targets of indirect control transfer instructions is desirable in several applications such as control-flow integrity. However, this problem is, in general, undecidable, which means that we can only hope for approximate solutions, i.e. , either to overapproximate or under-approximate the actual set of targets. Nevertheless, the switch statement in $\mathrm{C} / \mathrm{C}++$ remains amenable to precise analysis. It is commonly implemented as an indirect jmp that is based on single variable indexing into a look-up table. This index variable is intra-procedurally bounded.

The analysis of jump tables enables us to (1) increase CFG precision, (2) instrument jump table data, and (3) avoid disassembly errors. The latter issue is relevant to architectures such as ARM where compilers inline jump table data in the code section. Fortunately, in x86-64, such data typically reside in a separate read-only section, which enables correct disassembly using linear sweep [4]

The analysis of jump tables can be challenging as compilers enjoy a lot of flexibility in implementing switch statements. A jump table can be control-bounded by checking the value of the index against a bound condition. Alternatively, should the expected values be dense, e.g., many values below 16, the compiler might prefer a data-bounded jump table, e.g, using a bitwise and with oxf. Additionally, compilers are free to divide a switch with many case labels into multiple jump tables. Our goal in this analysis is to recover information about each individual jump table. This includes its control flow targets and total number of entries.

We propose sliced microexecution, a novel method for jump table analysis which combines classical backward slicing with microexecution [19]. The latter refers to the ability to emulate any code fragment without manual inputs. Basically, for each indirect jmp in a function, bcov attempts to test the sequence of hypotheses depicted in Table 2. If they are invalid then bcov aborts the analysis and considers this jmp to be a tail-call. Otherwise, bcov proceeds with the actual recovery depending on the type of jump table which can generally either be control-bounded or data-bounded.

We discuss this method based on the example shown in Figure 4. First, bcov has to test hypothesis \#1 by backward slicing from $0 \times 9 f 719$ until it reaches instruction at $0 \times 9 f 712$ which has a memory dependency. This dependency has a base address in $r 15$.

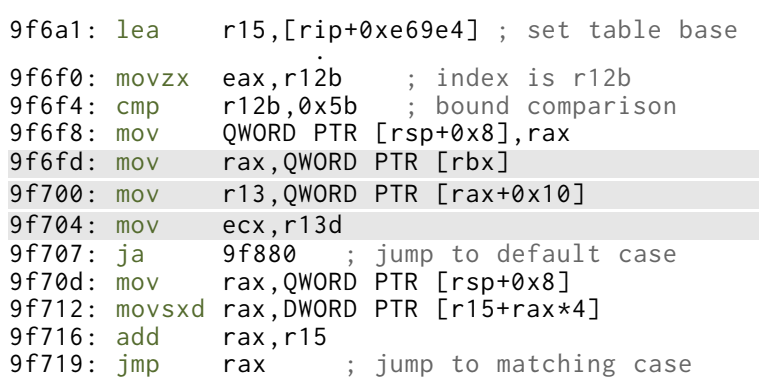

Figure 4: Jump table example from perl v5.28 compiled with gcc v7.3. Highlighted instructions are not part of the backward slice. The jump table base is set relatively far at $0 \times 9 \mathrm{f} 6 \mathrm{a} 1$.

So is this base address constant? Backward slicing for $r 15$ shows that it is constant indeed. Note that a jump table should depend on a single variable used as the index. The table's base address is a constant determined at compile time.

We move now to test hypothesis \#2. It is tested by spawning a condition slicer upon encountering each conditional jmp, .e.g, instruction at $0 \times 9 \mathrm{f} 707$. This slicer is used to check whether the variable influencing the bound condition is also the jump table index. This is the case in our example at $\theta \times 9 f 6 f \theta$ where the value in $\mathrm{r} 12 \mathrm{~b}$ influences both the condition at $0 \times 9 \mathrm{f} 707$ and the jump table index. Now that a bound condition is found we need to test it against hypothesis \#3.

A jump table might be preceded by multiple conditional comparisons that depend on the index. We apply heuristics to quickly discard the ones that can not represent a bound condition, e.g., comparisons with zero. However, there can still be more than one candidate. Here, we leverage the fact that a bound condition should dominate the jump table. Otherwise, a path in CFG would exist where the index value remains unbounded. We check for dominance during the backward CFG traversal needed for slicing. Basically, it should not be possible to bypass the bound condition.

Backward slicing produces a program slice (code fragment) which captures the essential instructions affecting the jump table. This slice represents a univariate block-box function with the index as its input variable. Modifying the index should trigger behavioral changes especially in the observed jump address at the output. Assuming that this slice represents a jump table, we reason about its behavior using microexecution. Also, we try to validate our assumption by widely varying the index.

Before microexecuting a slice, bcov first loads the binary using a built-in ELF loader. Then, it initializes a valid memory environment for the given program slice. For example, it allocates memory for the pointer $[r s p+0 \times 8]$ and assigns a valid address to $r s p$. It is now possible to start "fuzzing" the index. However, the expected behavior of the slice depends on the type of jump table.

In control-bounded jump tables, a change in behavior must be observed in the intervals $[0, b)$ and $(b,+\infty)$ where $b$ is the bound constant. This constant is located in the first instruction that sets the flags before the bound condition. In our example, this is the instruction at $0 \times 9 \mathrm{f} 6 \mathrm{f} 4$. bcov tests 24 index values in total, 8 of which are sampled from $[0, b]$ including $0, b-1$, and $b$. The remaining 16 values increase exponentially, in powers of 2 , starting from $b+1$. We found this scheme to give us high confidence in the results. 
The jump table is expected to target an instruction inside the current function for most inputs in $[0, b)$. On the other hand, the jump table should not be reachable for all inputs in $(b,+\infty)$. That is, the bound condition should redirect control flow to the default case. Should the behavior of the program slice not match what we expect from a control-bounded jump table, then we abort and assume that it is data bounded. Note that we are not strict about the behavior for input $b$ since the bound condition might check for equality.

Assuming that a given indirect jmp represents a data-bounded jump table, we need effective techniques to (1) stop backward slicing, (2) validate our assumption, and (3) explore the bound limits. Note that compilers might use more than one bitwise instruction to bound the index. Moreover, developers might prefer computed gotos over switch statements. ${ }^{2}$ In this case, they need to assume responsibility for checking index bounds.

To cope with this implementation diversity, bcov continues backward slicing as long as the current slice depends on only one variable. For example, assume that rax holds the index and is later used as a base register to read from memory. This means that the current slice would depend on rax as well as the variable accessed in memory. Backward slicing would stop before this increase in dependencies. Then, bcov executes the program slice 24 times, each time increasing the index exponentially while setting the least significant bits to one. This allows us to explore the bound limits in the common case of bitwise and with a bitmask like $0 x f$. Other bit patterns are also tried to better penetrate combinations of bitwise instructions. Our key insight is that we should not have full control over the jump target. That is, arbitrary change in the index should be reflected in a constrained change in the jump target. Additionally, jump targets need to be located in the current function similar to the case of control-bounded jump tables. Should the program slice withstand these diverse tests, then we can be highly confident that it represents a jump table.

Our evaluation shows that sliced microexecution is precise and robust against various compiler optimizations. It allowed bcov to reliably recover the jump tables in the core loop of the Python interpreter, located in function_PyEval_EvalFrameDefault. Note that these jump tables are compiled from complex computed gotos.

\section{STATIC INSTRUMENTATION}

In this section, we first consider a strategy to reduce instrumentation overhead by carefully selecting a basic block to probe in a superblock. Then, we discuss handling short basic blocks by means of hosting their detours in larger neighboring basic blocks.

\subsection{Optimized Probe Selection}

Generally, probing a $\mathrm{BB}$ requires inserting a detour targeting its designated trampoline. A detour occupies 5 bytes and can either be a direct jmp or call. Consequently, one or more original instructions must be relocated to the trampoline. This relocation overhead varies due to the instruction-size variability in x86-64. Note that a pcrelative mov, which occupies 7 bytes, represents an unavoidable overhead for updating coverage data in each trampoline. Hence, our goal is to reduce the relocation overhead.

\footnotetext{
${ }^{2}$ Computed gotos is a gcc extension to $\mathrm{C}$ which is also supported in clang.
}

Table 3: BB classification used in probe selection. Types are shown in ascending order based on expected relocation overhead. The terms long and short are relative to detour size ( 5 bytes). Short types require relocating preceeding $(\mathrm{RP})$ instruction(s).

\begin{tabular}{lll} 
Type & RP & Relocation overhead \\
\hline return & maybe & Can be only 1 byte depending on the padding \\
long-jump & no & Size of jmp instruction which is $\geq 5$ bytes \\
long-call & no & Size of call instruction which is $\geq 5$ bytes \\
jump-tab & no & Size of jmp instruction to original code (5 bytes) \\
short-call & yes & Similar to long-call but with RP overhead added \\
short-jump & yes & Similar to long-jump but with RP overhead added \\
internal & maybe & Size of relocated instruction(s) inside the BB \\
long-cond & no & Rewriting incurs a fixed 11 byte overhead \\
short-cond & yes & Similar to long-cond but with RP overhead added \\
\hline
\end{tabular}

To this end, we iterate over all BBs in a superblock and select the one expected to incur the lowest overhead. First, we have to establish whether a detour can be accommodated in the first place. A BB that satisfies $s+p<5$ is considered a guest, where $s$ and $p$ are the byte size and padding size respectively. A superblock that contains only guest BBs is handled via detour hosting (§5.2). Now we examine the type and size of the last instruction of each $\mathrm{BB}$ and whether the $\mathrm{BB}$ is targeted by a jump table. These parameters are translated to the types depicted in Table 3. These BB types are organized in a total order. This means, for example, we strictly prefer a long-call over a long-cond should both exist in the same superblock. This type order is primarily derived from empirical observation. However, we did not necessarily experiment with all possible combinations. Preferring long-call over short-call should be intuitive. The latter incurs an additional overhead for relocating at least one instruction preceding the call.

We observed that return basic blocks are usually padded (55\% on average). Padding size is often more than 3 bytes which translates to a relocation overhead of only one byte - the size of a ret instruction. Also, favoring long-jmp over long-call provided around 3\% improvement in both relocation and performance overheads. On the other hand, short-call had only a slight advantage over shortjmp. This might be due to the fixed 2-byte size of the latter, which leads to relocating more instructions. However, our experiments were not always conclusive, e.g., between jump-tab and short-call.

Relocating an instruction depends on its relation to the PC (called rip in x86-64). Position-independent instructions can simply be copied to the trampoline. However, we had to develop a custom rewriter for position-dependent instructions. The rewriter preserves the exact semantics of the original instruction whether it explicitly or implicitly depends on rip. For example, a long-cond instruction will be rewritten in the trampoline to a matching sequence consisting of a long-cond ( 6 bytes) and a jmp ( 5 bytes).

Jump table instrumentation has the unique property of preserving the original code. It is a data-only mechanism that enables us to probe even one-byte BBs. However, for it to be applicable, a $\mathrm{BB}$ has to be targeted by a patchable jump table. A jump table is patchable if its entries are either 32-bit offsets or absolute addresses. We observed that about $92 \%$ of more than 46,000 jump tables in our 
dataset are patchable. In fact, we found that 8-bit and 16-bit offsets are only used in libopencv_core.

Finally, our probe selection strategy is effective in reducing relocation overhead. However, it is not necessarily optimal. We observed high variance in the padding of BBs of type return, i.e., return is not always the best choice. Also, instrumenting a loop head can unnecessarily trigger multiple coverage updates. A loopaware strategy might reduce performance overhead by choosing a BB outside the loop as an alternative. Such optimizations are left for future work.

\subsection{Detour Hosting}

The instruction-size variability in $\mathrm{x} 86-64$ suggests that some BBs are simply too short to safely insert a detour without overwriting other BBs. In our dataset, we found that about $7 \%$ of all BBs are short (size $<5$ bytes). Left without a probe, we risk losing coverage information of a particular short BB and, potentially, all of its dominators. One possible solution is to relocate the entire function to a larger memory area. However, this is costly in terms of code size and the engineering effort required to fix relocated code references. For example, throwing an exception from a relocated function without fixing its corresponding CFI record might lead to abrupt process termination.

The method adopted in bcov is detour hosting. It significantly reduces the relocation overhead while preserving the stability of code references at basic-block level. Here, the size of a guest BB needs to be at least 2 bytes, which is enough to insert a short detour targeting a reachable host $\mathrm{BB}$, i.e., within about \pm 128 bytes. The host $\mathrm{BB}$ must be large enough to accommodate two regular detours, i.e., at least 10 bytes. The first detour targets the host trampoline while the other detours would target the trampolines of their respective guests. Note that we can safely overwrite padding bytes of both the guest and host. Also, the host does not need to be entirely relocated. Relocating a subset of its instructions might be sufficient.

Figure 5 depicts a detour hosting example. It involves a guest BB consisting of a single indirect call ( 3 bytes). The tricky part about a call is that its return address must be preserved. A sub instruction ( 5 bytes) is used to adjust the return address in the trampoline from 0xad67fd to its original value of $0 x a d 6803$. This adjustment also clobbers the CPU flags which is safe since they are not preserved across function calls in the $\mathrm{x} 86-64 \mathrm{ABI}$. Note that this is the only case where we modify the CPU state.

Now we have the following allocation problem: given a guest $g$ and a set of suitable hosts $H=\left\{h_{1}, h_{2}, . ., h_{n}\right\}$, find the host $h_{i}$ whose selection incurs minimal overhead. Also, we are interested in the more general formulation: given a set of guests $G=\left\{g_{1}, g_{2}, . ., g_{k}\right\}$ and a set of hosts $H=\left\{h_{1}, h_{2}, \ldots, h_{n}\right\}$, where each host is suitable for at least one guest, find a function mapping $M: G \rightarrow H$ such that the overhead is minimal. We approach this problem using a greedy strategy where we prefer, in this order, (1) packing more guests in a single host, (2) a host already selected for instrumentation over an otherwise intact host, (3) a host that is closer to the guest. Basically, for each guest, we iterate over all reachable BBs. A BB can offer a hosting offset, if possible. A higher offset means that more guests are packed in this host. The initial offset is 5 bytes from the start of the host. Should the offered offsets be equal, we look into (2) to

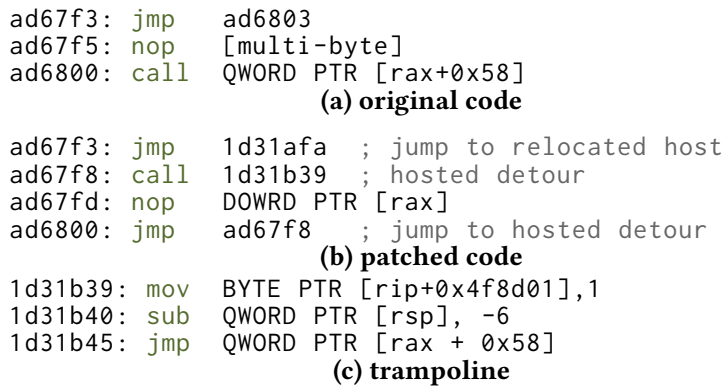

Figure 5: Detour hosting example taken from Ilc v8.0 compiled with clang v5.0. (a) host is a short $\mathrm{jmp}$ at $0 \times \mathrm{xad} 67 \mathrm{f} 3$ followed by 11 padding bytes. (b) inserting 2 detours leaves 3 padding bytes. (c) return address adjusted at $0 \times 1 \mathrm{~d} 31 \mathrm{~b} 40$. Original call at $0 \times a d 6800$ is rewritten to a matching jmp at $0 \times 1 \mathrm{~d} 31 \mathrm{~b} 45$

avoid, as much as possible, relocating otherwise intact BBs. Finally, should both (1) and (2) be equal, then we look into (3) to improve the code cache locality.

It is not possible to probe one-byte guests. Also, a suitable host might not be found. However, we can still reduce the loss in coverage information in such cases. To this end, we try to probe all of the immediate predecessors of the current $\mathrm{SB}$, containing the guest, in the SB-DG. However, this does not necessarily entail adding more probes. For example, additional probes are unnecessary in the leaf-node policy should the current SB have siblings in the SB-DG. In the any-node policy, on the other hand, the predecessors might be probed already.

Our detour hosting strategy targets a sweet spot balancing performance and relocation overheads. It achieves a hosting ratio of 1.2 guests per host on average. Also, it was able of hosting up to 14 guests in a single host. Around $80 \%$ of the hosts are already probed. That is, relocation overhead is expected for them anyway. Finally, it allowed bcov to host about $94 \%$ of all the guests.

\section{IMPLEMENTATION}

We implemented our approach in the tool bcov. Our tool accepts an ELF module (executable or shared library) as input. It starts with a set of module-level analyses such as reading function definitions, parsing CFI records, and building the call graph. Our non-return analysis implementation is similar to [29]. We omit the details as they are not part of our core contribution.

Then, bcov moves to function-level analyses such as building the CFG (including jump tables), dominator trees, and superblock dominator graph. Probes are determined based on the instrumentation policy set by the user. bcov can be used for patching or coverage reporting. The latter mode requires a data file dumped from a patched module. The instrumentation policy used for coverage reporting must match the one used for patching.

We implemented the modern SEMI-NCA dominator tree algorithm [18] and Tarjan's classical SCC algorithm. We used capstone [11] for disassembly and implemented a wrapper around unicorn [40] for microexecution. In total, this required about 17,000 LoC in $\mathrm{C}++$ (testing code excluded). The run-time library bcov-rt is implemented in $\mathrm{C}$ in $\sim 250 \mathrm{LoC}$. 
Table 4: Selected evaluation subjects. Used recent package versions.

\begin{tabular}{llll} 
Module & Package & Lang. & Domain \\
\hline gas & binutils-2.32 & $\mathrm{C}$ & Assemblers \\
perl & perl-5.28.1 & $\mathrm{C}$ & Interpreters \\
python & cpython-3.7.3 & $\mathrm{C}$ & Interpreters \\
libMagickCore & ImageMagick-7.0.8 & $\mathrm{C}$ & Image processing \\
ffmpeg & FFmpeg-4.1.3 & $\mathrm{C}$ & Video processing \\
libxerces-c-3.2 & xerces-c-3.2.2 & $\mathrm{C}++$ & XML processing \\
libopencv_core & opencv-4.0.1 & $\mathrm{C}++$ & Computer vision \\
Ilc & llvm-8.0.0 & $\mathrm{C}++$ & Compilers \\
\hline
\end{tabular}

\section{EVALUATION}

Our evaluation is guided by the following research questions:

RQ1 Can bcov transparently scale to large real-world binaries? RQ2 What is the instrumentation overhead in terms of performance, memory, and file size?

RQ3 Have we pushed the state of the art in jump table analysis? RQ4 To what extent can bcov provide better efficiency in comparison to its direct alternatives, namely, DBI tools?

RQ5 Can bcov accurately report binary-level coverage?

For evaluation, we selected eight modules from popular opensource packages offering diverse functionality. They are summarized in Table 4 . We compiled each module using four compilers in three different build types. Specifically, we used the compilers gcc5.5, gcc-7.4, clang-5.0, and clang-8.0. This gives us a representative snapshot of the past three years of developments in gcc and clang respectively. The build types are debug, release, and Ito. The latter refers to link-time optimizations. Compiler optimizations were disabled in debug builds and enabled in release and Ito builds. Enabled optimizations depend on the default options of their respective package which can be at levels 02 or 03 .

This results in 12 versions of each module and a total of 95 binaries. ${ }^{3}$ Our tool was capable of patching 88 binaries without modifying the build system. However, we had to modify the linker script in 7 instances where relocating ELF segment headers was not possible. We instructed the linker to leave 112 bytes, which is enough for our segment headers, after the original segment headers. This change is small affecting only one line in the linker script. The bcov-rt runtime was injected using the LD_PRELOAD mechanism. All experiments were conducted on an Ubuntu 16.04 PC with Intel ${ }^{\circledR}$ i7-6700 CPU and 32GB of RAM.

\section{RQ1. Scalability and Transparency}

Our choice of subjects directly supports our claim regarding scalability. Figure 6 shows a comparison in terms of code size relative to objdump, a commonly used subject in binary analysis research. Code size is measured using the popular size utility. Note that bcov can analyze and patch our largest subject, Ilc, in $\sim 30$ seconds. In our experiments, we used bcov to instrument all functions available in the . text section. More than $1.6 \times 10^{6}$ functions have been instrumented across 95 binaries. The policies leaf-node and any-node have been applied separately, i.e., subjects were instrumented twice.

\footnotetext{
${ }^{3}$ Compiling Ilc with gcc-5.5 in Ito build resulted in a compiler crash.
}

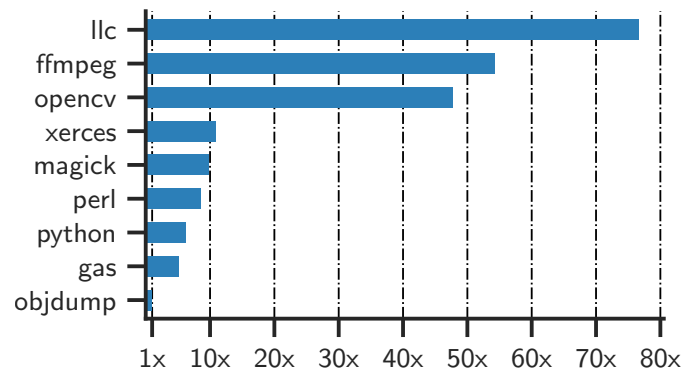

Figure 6: Comparing the code size of our subjects to objdump (code size about $339 \mathrm{~KB}$ ). Code size reported with GNU size utility.

Transparency is important in coverage instrumentation. This practically means that bcov should not introduce test regressions. We evaluated this criterion by replacing original binaries with instrumented versions and re-running their test suites. Our instrumentation did not introduce any regressions despite the fact that (1) we systematically patch all functions, even compiler-generated ones, and (2) our benchmark packages include extensive test suites. For example, the perl test suite runs over one million checks.

\section{RQ2. Instrumentation Overhead}

Figure 7 depicts the instrumentation overhead of the any-node policy relative to the original binaries. We omit the detailed evaluation of the leaf-node policy because of the lack of space. The average performance overheads of the leaf-node and any-node polices are $8 \%$ and $14 \%$ respectively. The overhead is measured based on the wall-clock time required to run individual test suites, .e.g, run "make test" to completion. This covers the overhead associated with instrumentation and dumping coverage data to disk. The latter overhead varies depending on the number of processes spawned during testing.

For example, all opencv tests are executed within a single process that dumps coverage data only once. On the other hand, unit testing of Ilc spawns over 7,500 processes in about 40s. This results in dumping $\sim 4 \mathrm{~GB}$ of coverage data which significantly contributes to the overall delay. Online merging of coverage data might reduce this disk IO overhead. To give a better intuition, we note that without online merging, llvm-cov would dump over $320 \mathrm{~GB}$ of coverage (and profiling) data for the same benchmark.

To gain a better insight into the distribution of performance overhead, we experimented with the following variants of the anynode policy:

- Detour only (DO). Here, we enabled only inserting detours and relocating overwritten instructions to the trampolines.

- Detour hosting (DH). Similar to DO, but we also enabled detour hosting for short basic blocks.

- Coverage update (CU). Similar to DH, but we also enabled inserting coverage update code in the trampoline.

Note that the last variant is equivalent to the any-node policy. We separately used each variant to patch all subjects in 4 different release builds. Then, we ran their respective test suites to measure the performance overhead. Interestingly, DO alone accounts for about $96 \%$ of the overhead on average. That is, DH and CU contributed only marginally. This result suggests that inlining coverage 


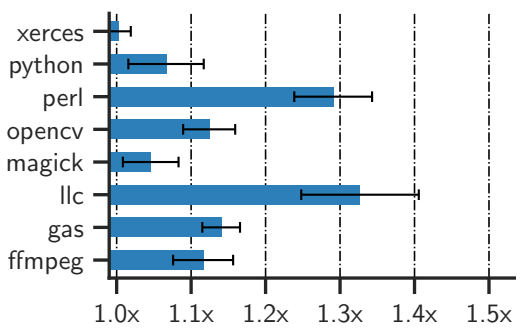

(a) performance overhead

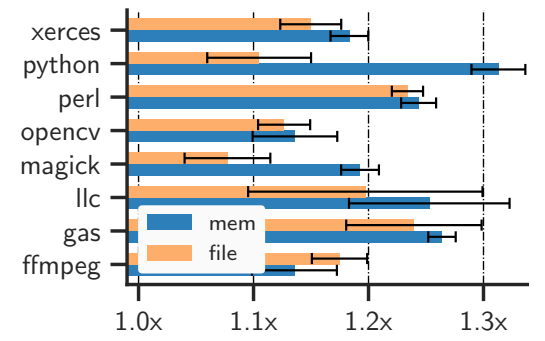

(b) size overhead

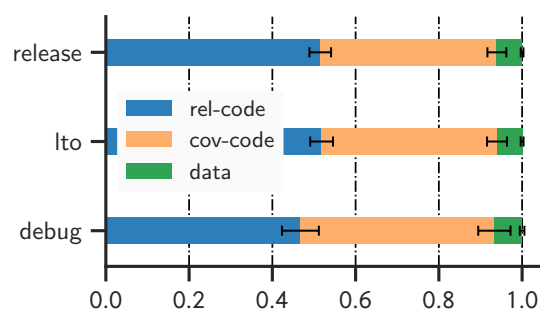

(c) overhead distribution

Figure 7: Overhead of the any-node instrumentation policy. (a) performance overhead accounts for instrumentation and dumping coverage data, (b) memory and file size overhead (c) distribution of memory overhead between code (relocated and coverage update) and coverage data.

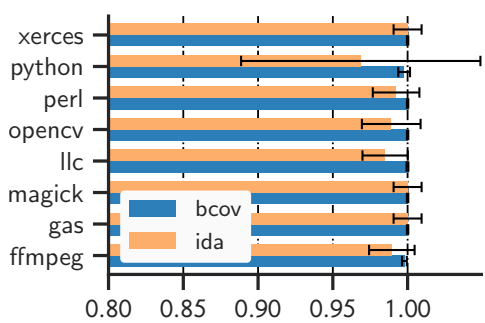

(a) clang binaries

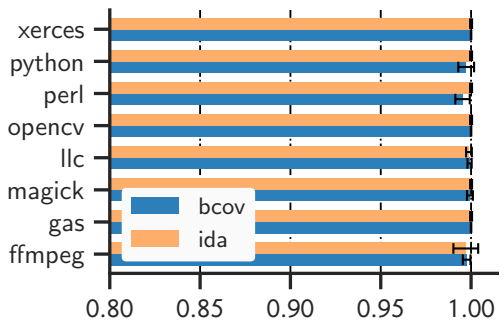

(b) gcc binaries

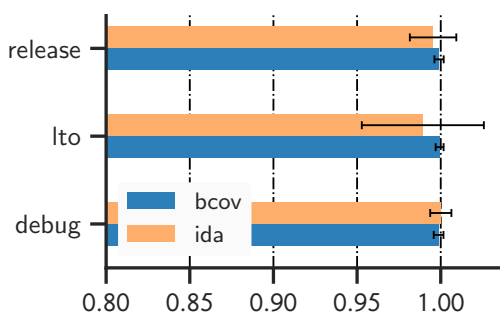

(c) build type

Figure 8: Normalized jump table analysis results in comparison to IDA Pro. (a) IDA Pro shows significant variance on clang binaries. (b) both tools are comparable on gcc binaries. (c) varying the build type did not affect bcov.

update code, and thereby eliminating the need for trampolines, may lead to substantial performance savings.

The average memory and file size overheads introduced by bcov are $22 \%$ and $16 \%$ respectively. We measure the memory overhead relative to the size of loadable ELF segments only. Recall that bcov does not affect the run-time heap or stack. The fact that other static instrumentation techniques need to duplicate the code segment $[3,26]$, suggests that the overhead of bcov is reasonable. Coverage data represents only $6 \%$ of the memory overhead. It is worth noting that compiler optimizations can force bcov to relocate more instructions. This might be due to emitting smaller basic blocks. However, our static instrumentation techniques are effective in reducing the difference in relocation overhead between debug and optimized builds as shown in Figure 7c.

\section{RQ3. Jump Table Analysis}

Evaluating sliced microexecution requires comparing bcov with representative binary analysis tools. However, it was not possible to compare with BAP [10] and angr [36] which are the leading academic tools. BAP does not have built-in support for jump table analysis, while angr (v8.18.10) crashed on opencv and Ilc binaries. For the remaining binaries, angr reported significantly fewer jump tables compared to IDA Pro. Therefore, we compare bcov only with IDA Pro (v7.2). This should not affect our results since IDA Pro is the leading industry disassembler.

Next, we have to establish the ground truth of jump table addresses - specifically, the addresses of their indirect jmp instructions. This is challenging as compilers do not directly emit such information. Therefore, we conducted a differential comparison. We observed that bcov and IDA Pro agree on the majority of jump tables including their targets, so we manually examined the remaining cases where they disagree. Both tools did not report false positives. That is, they only missed jump tables. This is expected in bcov as repeated microexecution inspires high confidence in its results. Therefore, our ground truth is the union of jump table addresses recovered by both tools.

Figure 8 depicts the recovery percentages relative to this ground truth consisting of over 46,000 jump tables. We control for different factors affecting compilation. We observed that IDA Pro delivers lower accuracy on clang binaries compared to gcc binaries, and its accuracy was affected by compiler optimizations. In comparison, bcov demonstrates higher robustness across the board.

\section{RQ4. Comparison with DBI Tools}

Pin and DynamoRIO (DR) are the most popular DBI tools. Both act as a process virtual machine which instruments programs while JIT-emitting instructions to a code cache. This complex process creates the following sources of overhead: (1) JIT optimization, and (2) client instrumentation. To evaluate this overhead on our test suites, we installed the latest stable releases of both tools, namely, Pin v3.11 and DR v7.1. We then replaced each of our subjects with a wrapper executable. In the case of shared libraries, we replaced their test harness with our wrapper. The test system would now run our wrapper, which in turn runs its corresponding original binary but under the control of a DBI tool. The wrapper reads a designated environment variable to choose between Pin and DR.

Figure 9 depicts the performance overhead of Pin and DR without client instrumentation. It also shows the overhead of DR after enabling drcov, its code coverage client. Note that Pin does not have a similar coverage client built-in. The overhead is measured relative to original binaries and is averaged for four different release builds. 
Table 5: Evaluating the accuracy of bcov based on drcov traces. We show the number of processes spawned during testing, corresponding dump sizes in $\mathrm{MB}$, and the total number of BBs and their instructions in original binaries. Both tools dump one coverage file per process. For each subject, we list the average/maximum of true positives (TP). FPs and FNs are also considered by listing the average precision and recall respectively. DR could not complete the test suite runs of perl and python. Omitted opencv as drcov's data was invalid due to a bug.

\begin{tabular}{|c|c|c|c|c|c|c|c|c|c|}
\hline Module & process \# & drcov size & bcov size & BB & Inst. & TP BB & TP Inst. & Precision & Recall \\
\hline xerces & 80 & 12.34 & 4.32 & 116378 & 420096 & 9523.2 / 21927 & 40651.2 / 92144 & $99,98 \%$ & $99.42 \%$ \\
\hline magick & 58 & 7.71 & 2.90 & 125521 & 521107 & $5689.4 / 20709$ & $21614.9 / 83444$ & $99,98 \%$ & $99.94 \%$ \\
\hline llc & 7862 & 3481.97 & 4176.16 & 1067151 & 4343021 & 45184.5 / 90952 & $257209.5 / 461656$ & $99,98 \%$ & $99.68 \%$ \\
\hline gas & 1235 & 71.94 & 38.56 & 60511 & 220447 & 2916.4 / 5015 & $11045.8 / 19578$ & $99.93 \%$ & $99.67 \%$ \\
\hline ffmpeg & 3309 & 423.45 & 762.39 & 496404 & 3050228 & $9682.0 / 14489$ & 41439.1 / 63591 & $99.98 \%$ & $99.94 \%$ \\
\hline
\end{tabular}

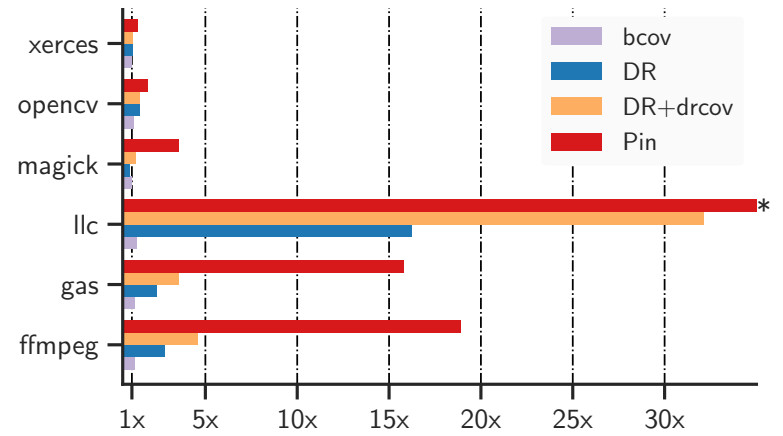

Figure 9: Comparing the performance overhead of Pin, DynamoRIO (DR), drcov, and bcov. Omitted perl and python as DR was unable of completing their test suite runs. $\left({ }^{*}\right)$ The actual overhead of Pin for Ilc is over 130x.

Both tools introduced regressions on perl and python. However, DR made tests hung on perl and crashed on the python test suite. This highlights the challenges of maintaining transparency in DBI tools. Note that the DBI overhead of executable subjects is significantly higher than that of shared libraries. This can be attributed to the start-up delay which dominates in short-running tests. The average performance overheads of Pin and DR are $29.1 \mathrm{x}$ and $4.1 \mathrm{x}$ respectively. Enabling drcov increases the average overhead to 7.3x. For the same benchmarks, bcov introduced an average overhead of $11 \%$ only. Our experiments show that bcov provides significantly better performance, transparency, and usability.

\section{RQ5. Coverage Report Accuracy}

We evaluate the accuracy of the reported coverage by tracing binaries that are instrumented by bcov. We use the any-node policy because of its precision. Note that comparing the coverage of original binaries separately to instrumented ones will likely introduce errors that are only caused by non-determinism. For example, repeatedly printing a simple: "Hello World" using perl will produce different instruction traces.

Initially, we obtained the ground truth traces using Intel PT (IPT). To this end, we collected about 2,000 sample tests from our test suites. Running these tests produces $104 \mathrm{~GB}$ of IPT data and $444 \mathrm{MB}$ of bcov coverage data. We used the standard perf tracing facilities in kernel v4.15 and later kernel v5.3. We tried many IPT configurations and restricted ourselves to tests terminating in $\leq 5$ seconds. Despite these efforts, we could not reliably evaluate bcov due to non-deterministic loss in IPT data. After all, disks might just be incapable of keeping up with the CPU [24].

We then turned to drcov to obtain the ground truth. This DR client dumps the address of encountered basic blocks (BB) heads, i.e., first instruction. We leverage the fact that our instrumentation does not modify BB heads. Based on this, we expect BBs reported as covered by bcov to appear in drcov's trace. We consider these BBs to be true positives (TP). On the other hand, a BB reported by bcov that was not found in the trace represents a false-positive (FP). Similarly, a false-negative (FN) is a tracked BB that was missed by bcov. Both FPs and FNs represent errors in the reported coverage. Our evaluation method is conservative given the potential overapproximation in the CFG. Also, we take into account the fact that drcov reports the heads of dynamic BBs. This means that should A and $\mathrm{B}$ be consecutive BBs where $\mathrm{A}$ is fallthrough, i.e., does not end with a branch, drcov might only report the head of A.

Our results are shown in Table 5. They are based on running the test suites of subjects compiled with gcc-7 in release build. The results are representative of other build types. The subjects are instrumented with bcov and also run under the control of DR's drcov. We list the average/maximum of TPs across all test processes. For example, the average number of TP BBs among 7,862 Ilc processes is $45,184.5$, and the maximum is 90,952 . The average precision and recall across all subjects are $99.97 \%$ and $99.95 \%$ respectively. This results in an average F-score of $99.86 \%$. Our evaluation suggests that the reported coverage errors are practically negligible. Nevertheless, there is still room for further improvement. Specifically, improving CFG precision and detour hosting can reduce FPs and FNs respectively.

\section{DISCUSSION}

In this section, we discuss potential issues and limitations of bcov.

RISC ISA. Inserting detours is generally easy in RISC ISAs thanks to their fixed instruction size. However, the addressing range can be significantly lower than the \pm 2 GB offered by $x 86-64$. Note that we patch each ELF module individually. This means that we only need an addressing range that is large enough to reach our patch code segment from the original code. For example, a range of $60 \mathrm{MB}$ would be sufficient for our largest subject. AArch64 offers a detour range of $\pm 128 \mathrm{MB}$ which can accommodate a large majority of binaries. AArch32 offers just $\sim 32 \mathrm{MB}$, in comparison. In such a case, a single detour instruction might not be sufficient. Additional options need to be investigated such as function relocation, literal pools, and modifications to linker scripts. 
In addition, we update coverage data using a single pc-relative mov which has a memory operand with a 32-bit offset. Generally, emulating the same functionality in RISC ISAs require more instructions and clobbering of registers. However, saving and restoring the clobbered registers is not always necessary. A liveness analysis can help us acquire registers with dead values. Similar analyses are already implemented in DBI tools.

Limitations and threats to validity. The precision of the recovered CFG can affect the coverage reported by our tool. While the implemented jump table and non-return analyses significantly increase CFG precision, they are still not perfect. Our prototype might miss jump tables, albeit only in a few situations. Also, while our experiments show that the non-return analysis in bcov is comparable to IDA Pro, both tools face the challenge of may-return functions. Such functions might not return to their caller depending on their arguments. Function Perl__force_out_malformed_utf8_message in perl is particularly noteworthy. In one binary, it is called 88 times (out of 89 total) with the argument die_here set, i.e, will not return. Developers can signal to the compiler that a particular call will not return using _-_builtin_unreachable(). Such hints are not available in the binary so we simply assume that all calls to may-return functions are returning. Consequently, bcov might spuriously report BBs following a may-return call as covered.

On another note, we believe that our subjects are representative of $\mathrm{C} / \mathrm{C}++$ software in Linux. However, generalizing our results to other native languages and operating systems requires further investigation. The simple mechanisms we use to implement detours and update coverage are also applicable to system software like kernel modules. However, special considerations might exist in such settings. Finally, our approach cannot be directly applied to dynamic code, e.g., self-modifying code.

\section{RELATED WORK}

Instrumentation using trampolines is known for a long time. It is typically used in restricted applications such as function interception [14]. We systematically use trampolines at a fine granularity to instrument individual basic blocks. Also, we are aggressive in exploiting padding bytes and hosting detours, which allows us to avoid relocating entire functions like in PEBIL [26].

Recently, several works considered static instrumentation via reassembly [15, 41] and recompilation [3]. Both enable instrumentation code to be inlined in their recovered artifacts, namely, assembly and IR respectively. Therefore, they are orthogonal to our approach, in principle. However, code inlining means that relocated references need to be fixed, e.g., in CFI records, which increases the engineering overhead. This can also be challenging to implement correctly since distinguishing references from scalars is an undecidable problem in general. In comparison, trampolines maintain reference stability which allowed us to seamlessly scale to large $\mathrm{C} / \mathrm{C}++$ binaries. Also, they make it easy to map analysis results back to the original binaries.

The analysis of jump tables was considered in several works. A combination of pattern matching and data-flow analysis was proposed in [7, 29]. Cifuentes et al. [12] use backward slicing to produce a slice, which they convert to a canonical IR expression before checking it against known jump table forms. A custom valueset analysis using SMT solving was implemented in JTR [13]. It is applied after lifting instructions to LLVM IR. In contrast, our approach, sliced microexecution, semantically reasons about jump tables without manual pattern matching. Also, it does not require lifting instructions to an IR. Such lifting is known to be errorprone [25] and can drastically slow down binary analyses [43]. Instead, we leverage the executable instruction semantics already available in off-the-shelf emulators. Moreover, we move beyond mere recovery to jump table instrumentation.

Tikir et al. [38] propose an approach for binary-level coverage analysis and use probe pruning to improve its efficiency. It is the closest related work to ours. However, our approaches differ in several aspects. First, they focus on dynamic coverage analysis where binaries can be analyzed, patched, and potentially restored at runtime. In contrast, our static instrumentation approach allows us to spend more time on optimizations. Second, their work builds on Dyninst [17], a generic binary instrumentation tool. However, the generality of Dyninst comes at a considerable cost in terms of overhead and complexity. For example, it has multiple levels of trampolines. In comparison, we focus on the bare minimum required for tracking code coverage. Consequently, bcov provides better performance and transparency. Finally, as acknowledged by the authors, the probe pruning technique implemented in bcov is more efficient than that of [38].

\section{CONCLUSION}

In this work, we presented bcov, a tool for binary-level coverage analysis. We implement a trampoline-based instrumentation approach and demonstrate that it can be both efficient and transparent while scaling to large $\mathrm{C} / \mathrm{C}++$ programs. However, this required an orchestrated effort where we leverage probe pruning, improve $\mathrm{CFG}$ precision, and cope with the instruction-size variability in x86-64 ISA. Our tool statically instruments ELF binaries without compiler support. It largely avoids the need to modify the build system and, consequently, allows for high usability. Also, we show that the produced coverage report is highly accurate, which can offer a valuable addition to the testing workflow. We make our tool and dataset publicly available to foster further research in this area.

\section{ACKNOWLEDGMENTS}

We would like to thank the anonymous reviewers of ESEC/FSE and USENIX Security for their insightful comments. Also, we would like to thank Fangrui Song for the helpful feedback. Deeban Babu provided assistance with BAP and angr experiments. This research has been funded by the federal state of Rhineland-Palatinate, Germany.

\section{REFERENCES}

[1] Hiralal Agrawal. 1994. Dominators, super blocks, and program coverage. In Proceedings of the 21st ACM symposium on principles of programming languages - POPL '94. ACM Press, New York, New York, USA, 25-34. https://doi.org/10. $1145 / 174675.175935$

[2] Paul Ammann and Jeff Offutt. 2016. Introduction to Software Testing. Cambridge University Press. https://doi.org/10.1017/9781316771273

[3] Kapil Anand, Matthew Smithson, Khaled Elwazeer, Aparna Kotha, Jim Gruen, Nathan Giles, and Rajeev Barua. 2013. A compiler-level intermediate representation based binary analysis and rewriting system. In Proceedings of 8th ACM European Conference on Computer Systems (EuroSys '13). ACM Press, Prague, Czech Republic, 295-308. https://doi.org/10.1145/2465351.2465380 
[4] Dennis Andriesse, Xi Chen, Victor van der Veen, Asia Slowinska, and Herbert Bos. 2016. An In-Depth Analysis of Disassembly on Full-Scale x86/x64 Binaries. In 25th USENIX Security Symposium. USENIX Association, Austin, TX, 583-600. https://www.usenix.org/conference/usenixsecurity16/technicalsessions/presentation/andriesse

[5] Dennis Andriesse, Asia Slowinska, and Herbert Bos. 2017. Compiler-Agnostic Function Detection in Binaries. In IEEE European Symposium on Security and Privacy - EuroS\ \&\}P'17. IEEE, 177-189. https://doi.org/10.1109/EuroSP.2017.11

[6] Tiffany Bao, Jonathan Burket, Maverick Woo, Rafael Turner, and David Brumley 2014. BYTEWEIGHT: Learning to Recognize Functions in Binary Code. In Proceeding of the 23rd USENIX Security Symposium. USENIX Association, San Diego, CA, 845-860. https://www.usenix.org/conference/usenixsecurity14/technicalsessions/presentation/bao

[7] M. Ammar Ben Khadra, Dominik Stoffel, and Wolfgang Kunz. 2016. Speculative disassembly of binary code. In International Conference on Compilers, Architecture and Synthesis for Embedded Systems - CASES'16. Pittsburgh, PA, USA. https: //doi.org/10.1145/2968455.2968505

[8] Marcel Böhme, Van-Thuan Pham, and Abhik Roychoudhury. 2016. Coveragebased Greybox Fuzzing as Markov Chain. In Proceedings of the 2016 ACM SIGSAC Conference on Computer and Communications Security - CCS'16. ACM Press, New York, New York, USA, 1032-1043. https://doi.org/10.1145/2976749.2978428

[9] Derek Bruening. [n.d.]. DynamoRIO: Dynamic Instrumentation Tool Platform. https://github.com/DynamoRIO/dynamorio

[10] David Brumley, Ivan Jager, Thanassis Avgerinos, and Edward J. Schwartz. 2011 BAP: A Binary Analysis Platform. In Computer Aided Verification (LNCS), Ganesh Gopalakrishnan and Shaz Qadeer (Eds.). Springer Berlin Heidelberg, 463-469. https://link.springer.com/chapter/10.1007/978-3-642-22110-1_37

[11] Capstone. [n.d.]. Multi-architecture disassembly framework. https://github com/aquynh/capstone

[12] C. Cifuentes and M. Van Emmerik. 1999. Recovery of jump table case statements from binary code. In Proceedings of the 7th International Workshop on Program Comprehension. IEEE Comput. Soc, 192-199. https://doi.org/10.1109/WPC.1999. 777758

[13] Lucian Cojocar, Taddeus Kroes, and Herbert Bos. 2017. JTR: A Binary Solution for Switch-Case Recovery. In International Symposium on Engineering Secure Software and Systems - ESSoS'17. Springer, Cham, 177-195. https://doi.org/10.1007/978-3319-62105-0 12

[14] Detours. [n.d.]. a software package for monitoring and instrumenting API calls on Windows. https://github.com/microsoft/Detours

[15] Sushant Dinesh, Nathan Burow, Dongyan Xu, and Mathias Payer. 2020. RetroWrite: Statically Instrumenting COTS Binaries for Fuzzing and Sanitization. In IEEE International Symposium on Security and Privacy - S\&P'20.

[16] DO178C. [n.d.]. Software Considerations in Airborne Systems and Equipment Certification. https://www.rtca.org

[17] Dyninst. [n.d.]. Tools for binary instrumentation, analysis, and modification. https://github.com/dyninst/dyninst

[18] Loukas Georgiadis. 2005. Linear-Time Algorithms for Dominators and Related Problems. Ph.D. Dissertation. Princeton University. https://www.cs.princeton. edu/research/techreps/TR-737-05

[19] Patrice Godefroid. 2014. Micro execution. In Proceedings of the 36th International Conference on Software Engineering - ICSE'14. ACM Press, New York, New York, USA, 539-549. https://doi.org/10.1145/2568225.2568273

[20] Rahul Gopinath, Carlos Jensen, and Alex Groce. 2014. Code coverage for suite evaluation by developers. In Proceedings of the 36th International Conference on Software Engineering - ICSE 2014. ACM Press, New York, New York, USA, 72-82. https://doi.org/10.1145/2568225.2568278

[21] Laura Inozemtseva and Reid Holmes. 2014. Coverage is not strongly correlated with test suite effectiveness. In Proceedings of the 36th International Conference on Software Engineering - ICSE'14. 435-445. https://doi.org/10.1145/2568225.2568271

[22] ISO-26262. [n.d.]. Road vehicles - Functional safety - Part 6: Product development at the software level. https://www.iso.org/obp/ui/\#iso:std:iso:26262:-1:ed-2:v1:en

[23] Marko Ivankovic, Goran Petrovic, René Just, and Gordon Fraser. 2019. Code coverage at Google. In Proceedings of the 2019 27th ACM Joint Meeting on European Software Engineering Conference and Symposium on the Foundations of Software Engineering - ESEC/FSE'19. 955-963.
[24] Linux Kernel. [n.d.]. Intel Processor Trace Documentation. https://github.com/ torvalds/linux/blob/master/tools/perf/Documentation/perf-intel-pt.txt

[25] Soomin Kim, Markus Faerevaag, Minkyu Jung, Seungll Jung, DongYeop Oh, JongHyup Lee, and Sang Kil Cha. 2017. Testing intermediate representations for binary analysis. In 32nd IEEE/ACM International Conference on Automated Software Engineering - ASE'17. IEEE, 353-364. https://doi.org/10.1109/ASE.2017. 8115648

[26] Michael A. Laurenzano, Mustafa M. Tikir, Laura Carrington, and Allan Snavely. 2010. PEBIL: Efficient static binary instrumentation for Linux. In IEEE International Symposium on Performance Analysis of Systems \& Software - ISPASS'10. IEEE, 175-183. https://doi.org/10.1109/ISPASS.2010.5452024

[27] Vu Le, Mehrdad Afshari, and Zhendong Su. 2014. Compiler validation via equivalence modulo inputs. In Proceedings of the 35th Conference on Programming Languages Design and Implementation - PLDI'14. ACM, 216-226. https: //doi.org/10.1145/2666356.2594334

[28] LibFuzzer. [n.d.]. a library for coverage-guided fuzz testing. https://llvm.org/ docs/LibFuzzer.html

[29] Xiaozhu Meng and Barton P. Miller. 2016. Binary code is not easy. In Proceedings of the 25th International Symposium on Software Testing and Analysis - ISSTA'16. ACM Press, New York, New York, USA, 24-35. https://doi.org/10.1145/2931037.2931047

[30] OSS-Fuzz. [n.d.]. continuous fuzzing of open source software. https://github. com/google/oss-fuzz

[31] Spencer Pearson, Jose Campos, Rene Just, Gordon Fraser, Rui Abreu, Michael D. Ernst, Deric Pang, and Benjamin Keller. 2017. Evaluating and Improving Fault Localization. In IEEE/ACM 39th International Conference on Software Engineering - ICSE'17. IEEE, 609-620. https://doi.org/10.1109/ICSE.2017.62

[32] Pin. [n.d.]. A Dynamic Binary Instrumentation Tool. https://software.intel.com/ en-us/articles/pin-a-dynamic-binary-instrumentation-tool

[33] Sanjay Rawat, Vivek Jain, Ashish Kumar, Lucian Cojocar, Cristiano Giuffrida, and Herbert Bos. 2017. VUzzer: Application-aware Evolutionary Fuzzing. In Network and Distributed System Security Symposium - NDSS'17. https://www.vusec.net/ download/?t=papers/vuzzer_ndss17.pdf

[34] SanitizerCoverage. [n.d.]. LLVM coverage instrumentation. https://clang.llvm. org/docs/SanitizerCoverage.html

[35] Sergej Schumilo, Cornelius Aschermann, Robert Gawlik, Sebastian Schinzel, and Thorsten Holz. 2017. kAFL: hardware-assisted feedback fuzzing for OS kernels. In Proceedings of the 26th USENIX Security Symposium. USENIX Association, 167-182. https://dl.acm.org/citation.cfm?id=3241204

[36] Yan Shoshitaishvili, Ruoyu Wang, Christopher Salls, Nick Stephens, Mario Polino, Andrew Dutcher, John Grosen, Siji Feng, Christophe Hauser, Christopher Kruegel, and Giovanni Vigna. 2016. SOK: (State of) The Art of War: Offensive Techniques in Binary Analysis. In IEEE Symposium on Security and Privacy - S\&P'16. IEEE, 138-157. https://doi.org/10.1109/SP.2016.17

[37] Robert Swiecki. [n.d.]. Honggfuzz: a security oriented fuzzer. https://github. com/google/honggfuzz

[38] Mustafa M. Tikir and Jeffrey K. Hollingsworth. 2002. Efficient instrumentation for code coverage testing. In Proceedings of the international symposium on Software testing and analysis - ISSTA '02, Vol. 27. ACM Press, New York, New York, USA, 86. https://doi.org/10.1145/566172.566186

[39] Paul Turner. [n.d.]. Retpoline: a software construct for preventing branch-targetinjection. https://support.google.com/faqs/answer/7625886

[40] Unicorn. [n.d.]. CPU emulator framework. https://github.com/unicorn-engine/ unicorn

[41] Shuai Wang, Pei Wang, and Dinghao Wu. 2015. Reassembleable disassembling. Proceedings of the 24th USENIX Security Symposium (2015), 627-642. https: //www.usenix.org/node/190921

[42] S. Yoo and M. Harman. 2010. Regression testing minimization, selection and prioritization: a survey. Software Testing, Verification and Reliability 22, 2 (mar 2010), n/a-n/a. https://doi.org/10.1002/stvr.430

[43] Insu Yun, Sangho Lee, Meng Xu, Yeongjin Jang, and Taesoo Kim. 2018. QSYM: A Practical Concolic Execution Engine Tailored for Hybrid Fuzzing. In 27th USENIX Security Symposium. 745-761. https://www.usenix.org/conference/ usenixsecurity18/presentation/yun

[44] Michal Zalewski. [n.d.]. Technical whitepaper for afl-fuzz. http://lcamtuf. coredump.cx/afl/technical_details.txt 